\title{
The magnetic field of IRAS 16293-2422 as traced by shock-induced $\mathrm{H}_{2} \mathrm{O}$ masers
}

\author{
F. O. Alves ${ }^{1}$, W. H. T. Vlemmings ${ }^{2}$, J. M. Girart ${ }^{3}$, and J. M. Torrelles ${ }^{4}$ \\ 1 Argelander-Institut für Astronomie, University of Bonn, Auf dem Hügel 71, 53121 Bonn, Germany \\ e-mail: falves@astro.uni-bonn.de \\ 2 Chalmers University of Technology, Onsala Space Observatory, 43992 Onsala, Sweden \\ e-mail: wouter.vlemmings@chalmers.se \\ 3 Institut de Ciències de l'Espai (IEEC-CSIC), Campus UAB, Facultat de Ciències, C5 par 2a , 08193 Bellaterra, Catalunya, Spain \\ e-mail: girart@ice.cat \\ ${ }^{4}$ Institut de Ciències de l'Espai (CSIC)-UB/IEEC, Universitat de Barcelona, Martí i Franquès 1, 08028 Barcelona, Spain \\ e-mail: torrelles@ieec.cat
}

Received 22 December 2011 / Accepted 30 March 2012

\begin{abstract}
Context. Shock-induced $\mathrm{H}_{2} \mathrm{O}$ masers are important magnetic field tracers of very high density gas. Water masers are found in both high- and low-mass star-forming regions, and are a powerful tool for comparing magnetic field morphologies in both mass regimes. Aims. We present one of the first magnetic field determinations for the low-mass protostellar core IRAS 16293-2422 at volume densities as high as $10^{8-10} \mathrm{~cm}^{-3}$. Our goal is to determine wether the collapsing regime of this source is controlled by magnetic fields or other factors such as turbulence.

Methods. We used the Very Large Array (VLA) to carry out spectropolarimetric observations of the $22 \mathrm{GHz}$ Zeeman emission from $\mathrm{H}_{2} \mathrm{O}$ masers. From the Stokes $V$ line profile, we are then able to estimate the magnetic field strength in the dense regions around the protostar.

Results. A blend of at least three maser features can be inferred from our relatively high spatial resolution data set $\left(\sim 0.1^{\prime \prime}\right)$, which is reproduced as a clear non-Gaussian line profile. The emission is very stable in terms of polarization fraction and position angle across the channels. The maser spots are aligned with some components of the complex outflow configuration of IRAS 16293-2422, and are excited in zones of compressed gas produced by shocks. The post-shock particle density is in the range of $1-3 \times 10^{9} \mathrm{~cm}^{-3}$, consistent with typical water-maser pumping densities. Zeeman emission is produced by a very strong line-of-sight magnetic field $(B \sim 113 \mathrm{mG})$.

Conclusions. The magnetic field pressure derived from our data is comparable to the ram pressure of the outflow dynamics. This indicates that the magnetic field is energetically important to the dynamical evolution of IRAS 16293-2422.
\end{abstract}

Key words. stars: formation - masers - polarization - ISM: magnetic fields - ISM: individual objects: IRAS 16293-2422

\section{Introduction}

Spectropolarimetric observations of masers are a powerful tool to study the magnetic field properties in the maser pumping zone. Water masers are unique because they are found in a variety of astrophysical environments and, in particular, starforming regions in distinct mass regimes. In contrast, methanol and $\mathrm{OH}$ masers are mostly found to be associated with highmass star formation sites (e.g., Vlemmings 2008; Sanna et al. $2010 \mathrm{a}, \mathrm{b})$. The most commonly observed water-maser line is the $\left(6_{16}-5_{23}\right)$ transition at $22 \mathrm{GHz}$, which is an excellent probe of molecular gas at very high volume densities $\left(n_{\mathrm{H}_{2}}\right.$ in the $10^{8}$ to $10^{10} \mathrm{~cm}^{-3}$ range, Elitzur et al. 1989). While the Zeeman splitting is small $\left(\sim 10^{-3} \mathrm{~Hz}_{\mu \mathrm{G}^{-1}}\right)$, the line strength is sometimes sufficient for measuring the circular polarization and therefore performing a direct measurement of the line-of-sight (LOS) component of the magnetic field. If linear polarization is also measured, then the full three-dimensional (3-D) magnetic field configuration can be derived.

There have been several recent studies of the efficiency of the maser spectropolarimetry technique. These have analyzed the magnetic field properties in very dense molecular environments around the circumstellar envelopes of evolved stars and young stars (e.g., Surcis et al. 2011a,b; Pérez-Sánchez et al. 2011). A representative illustration of how magnetic fields can be resolved on very small spatial scales was obtained by Vlemmings et al. (2006), who carried out Very Long Baseline Array (VLBA) polarization observations of $\mathrm{H}_{2} \mathrm{O}$ masers around the Cepheus $\mathrm{A}$ HW2 high-mass object.

Thermal dust polarization emission is difficult to observe in the most embedded portions of molecular clouds due to the low instrumental sensitivity to polarized data (and the feasibility is then limited toward a handful of objects). Submillimeter (submm) emission suffers from depolarization effects such as unresolved fields or the roundness of dust grains (Goodman et al. 1992, 1995; Lazarian et al. 1997). Water masers can be thought of as an observational tool to overcome this issue, since they are excited at very high densities.

IRAS 16293-2422 (hereafter, I16293) is a prototypical lowmass protostellar system located in the $\rho$ Ophiuchus molecular cloud ( $d \simeq 120 \mathrm{pc}$, Loinard et al. 2008). This source is a well-studied binary system, usually referred to as sources A and $\mathrm{B}$, that has an angular separation of 5" (600 AU) and is very 
embedded in a dense molecular core (Wootten 1989; Looney et al. 2000). Both sources have a very rich chemistry that is typical of that found in hot cores (Ceccarelli et al. 2000; Kuan et al. 2004; Bisschop et al. 2008; Jørgensen et al. 2011). Although component B appears to be a single source, high resolution interferometer data reveal that source A contains a higher degree of fragmentation. Two dust submm components were detected within this object with an angular separation of $\sim 0.6^{\prime \prime}$ ( 72 AU, Chandler et al. 2005). I16293 has two large-scale $\left(\sim 1^{\prime}\right)$ bipolar $\mathrm{CO}$ outflows, one of them associated with source $\mathrm{A}$, while the powering source of the other $\mathrm{CO}$ outflow is a matter of debate (Walker et al. 1988; Stark et al. 2004; Yeh et al. 2008). Observations of the $\mathrm{SiO}$ (8-7) emission have revealed that there is also a compact molecular outflow associated with source A (Rao et al. 2009). I16293 has strong water maser emission that has been well-monitored (Wilking \& Claussen 1987; Terebey et al. 1992; Claussen et al. 1996; Furuya et al. 2003). The strongest features appear at blueshifted and redshifted LSR velocities with respect to the ambient cloud velocity $\left(\sim 4 \mathrm{~km} \mathrm{~s}^{-1}\right)$, typically with $V_{\text {LSR }}$ between -5 and $10 \mathrm{~km} \mathrm{~s}^{-1}$. The $\mathrm{H}_{2} \mathrm{O}$ maser emission very often has intensities of more than $100 \mathrm{Jy}$, and at times it is stronger than $300 \mathrm{Jy}$, at LSR velocities of $\sim 7 \mathrm{~km} \mathrm{~s}^{-1}$.

Tamura et al. (1993) performed observations of the $1.1 \mathrm{~mm}$ dust polarized emission toward I16293 at an angular resolution of $19^{\prime \prime}$. They found that the magnetic field lines are perpendicular to the major axis of the dense elongated disk-like molecular structure $\left(\sim 10^{\prime \prime}\right.$ size, $\left.\sim 1200 \mathrm{AU}\right)$ observed in $\mathrm{C}^{18} \mathrm{O}$ by Mundy et al. (1990). This morphology was corroborated by Rao et al. (2009), who used the Submillimeter Array (SMA) and obtained a polarization map at an angular resolution of $\sim 2^{\prime \prime}(\sim 240 \mathrm{AU})$, resolving the sources $\mathrm{A}$ and $\mathrm{B}$. The mean volume density traced by the SMA maps is $\sim 6 \times 10^{7} \mathrm{~cm}^{-3}$. The polarization pattern around source $\mathrm{A}$ is compatible with a hourglass morphology for the magnetic field, whose strength was estimated to be $\sim 4.5 \mathrm{mG}$. The SMA maps show that there is a misalignment between the outflow direction and the magnetic field axis, that is roughly in agreement with model predictions where the magnetic energy is comparable to the centrifugal energy. In contrast, source B is associated with a uniform and apparently undisturbed magnetic field.

In this work, we report spectropolarimetric Very Large Array (VLA) observations of $\mathrm{H}_{2} \mathrm{O}$ masers toward I16293. In Sect. 2, we describe the details of the observational setup. In Sect. 3, the results obtained from the maser spectroscopy are shown. In Sect. 4, we discuss a possible correlation between the masers features and the outflows. The magnetic field strength estimation and its implications for the core dynamical evolution are discussed in Sects. 5 and 6, respectively. Finally, in Sect. 7 we summarize our conclusions.

\section{Observations}

Our observations were performed with the VLA $\left(\mathrm{NRAO}^{1}\right.$, New Mexico, USA) in its extended A-configuration, on 2007 June 25 and 27. Each observing track lasted $\sim 5.5 \mathrm{~h}$. A total of 27 antennas were used, 10 of which had already been retrofitted with the new system, resulting in a combined VLA/EVLA (extended VLA) observation. We used the $K$ band receivers $(22-24 \mathrm{GHz})$ tuned to the frequency of the water maser $\left(6_{16}-5_{23}\right)$ rotational transition $\left(v_{0}=22.23508 \mathrm{GHz}\right)$. We took

\footnotetext{
1 The National Radio Astronomy Observatory - NRAO - is a facility of the National Science Foundation, operated under cooperative agreement by Associated Universities, Inc.
}

advantage of the full polarization capability of the correlator, selecting a bandwidth of $0.7813 \mathrm{MHz}\left(\sim 10.5 \mathrm{~km} \mathrm{~s}^{-1}\right.$ in velocity). The spectral setup contains a total of 128 channels, which covers most of the velocity range of the strongest water maser features (observed around the brightest feature previously reported at $\sim 7 \mathrm{~km} \mathrm{~s}^{-1}$ ) at the spectral resolution of $0.08 \mathrm{~km} \mathrm{~s}^{-1}$. The quasar J1626-298 was used as the gain calibrator. The quasar $\mathrm{J} 1331+305$ and the radio source $\mathrm{J} 1751+096$ were used as polarization calibrators in order to correct both the instrumental feed polarization and the position angle of the polarization vectors. All three calibrators were also used for bandpass corrections. We performed self-calibration using the channel with the strongest intensity. Since the detected emission was unresolved, both phase and amplitude self-calibration solutions were applied to the other channels.

The combined VLA/EVLA setup introduces about $8 \%$ closure errors into the VLA-EVLA baselines-based corrections for the bandpass. This happens because of the distinctly different bandpass response of the EVLA antennas compared to the VLA ones. These errors become larger at narrow bandwidths, when the processing of the digital signal of EVLA antennas aliases the power response at the band edges. During the data reduction, we took special care to determine the bandpass solutions by performing the particular calibration strategies recommended to the user in the NRAO webpage ${ }^{2}$. They consisted of flagging EVLA-EVLA baselines prior to bandpass calibration and unflagging them prior to applying the bandpass solutions to the data set. The bandpass corrections are then applied to both an average single-channel multi-source dataset ("channel 0" file) and the multi-channel original file.

Data reduction was performed with the Astronomical Image Processing Software package (AIPS). Imaging of Stokes parameters $I, Q$, and $U$ were generated with a quasi-uniform weighting (robust $=-1$, Briggs 1995). Maps of polarized fraction $(P)$ and position of polarization angles (PA) were obtained by combining the Stokes $Q$ and $U$ images in such a way that $P=\frac{I_{P}}{I}=$ $\frac{\sqrt{Q^{2}+U^{2}}}{I}$ and PA $=\frac{1}{2} \tan ^{-1}\left(\frac{U}{Q}\right)$. The resulting synthesized beam is $0.14^{\prime \prime} \times 0.08^{\prime \prime}$, with a position angle of $-5.7^{\circ}$. The rms noise for channels where no emission was detected is $\sim 8 \mathrm{mJy}_{\text {beam }}{ }^{-1}$ and increases to $23 \mathrm{mJy}_{\text {beam }}{ }^{-1}$ at the peak emission channel. A slightly lower rms is observed for polarized intensity.

\section{Results}

\section{1. $\mathrm{H}_{2} \mathrm{O}$ maser line}

The contour channel maps of the $\mathrm{H}_{2} \mathrm{O}$ emission observed with the VLA/EVLA data are shown in Fig. 1. The emission extends over a wide range of velocities $\left(4.5<V_{\mathrm{LSR}}<9 \mathrm{~km} \mathrm{~s}^{-1}\right)$, i.e., at redshifted LSR velocities with respect to the cloud systemic velocity $\left(\sim 4 \mathrm{~km} \mathrm{~s}^{-1}\right)$. Our spectral setup covers only a small portion of LSR velocities blueward of it $\left(\sim 2<V_{\mathrm{LSR}}<4 \mathrm{~km} \mathrm{~s}^{-1}\right)$ but no emission was detected within this spectral range. The water maser line has a peak intensity of $170 \mathrm{Jy} \mathrm{beam}^{-1}$, which is detected at $V_{\mathrm{LSR}} \simeq 7.4 \mathrm{~km} \mathrm{~s}^{-1}$. This emission was also extensively reported in previous surveys as the brightest feature (see, for instance, Claussen et al. 1996; Furuya et al. 2003). Some channels have emission that is extended slightly eastward ( $V_{\mathrm{LSR}}$ between $\simeq 6.5$ and $7 \mathrm{~km} \mathrm{~s}^{-1}$ ), suggesting that the observed emission in these channels is marginally resolved.

\footnotetext{
2 http://www.vla.nrao.edu/astro/guides/evlareturn/ postproc/index. shtml\#closure-line
} 
F. O. Alves et al.: The magnetic field of IRAS $16293-242$ as traced by $\mathrm{H}_{2} \mathrm{O}$ masers

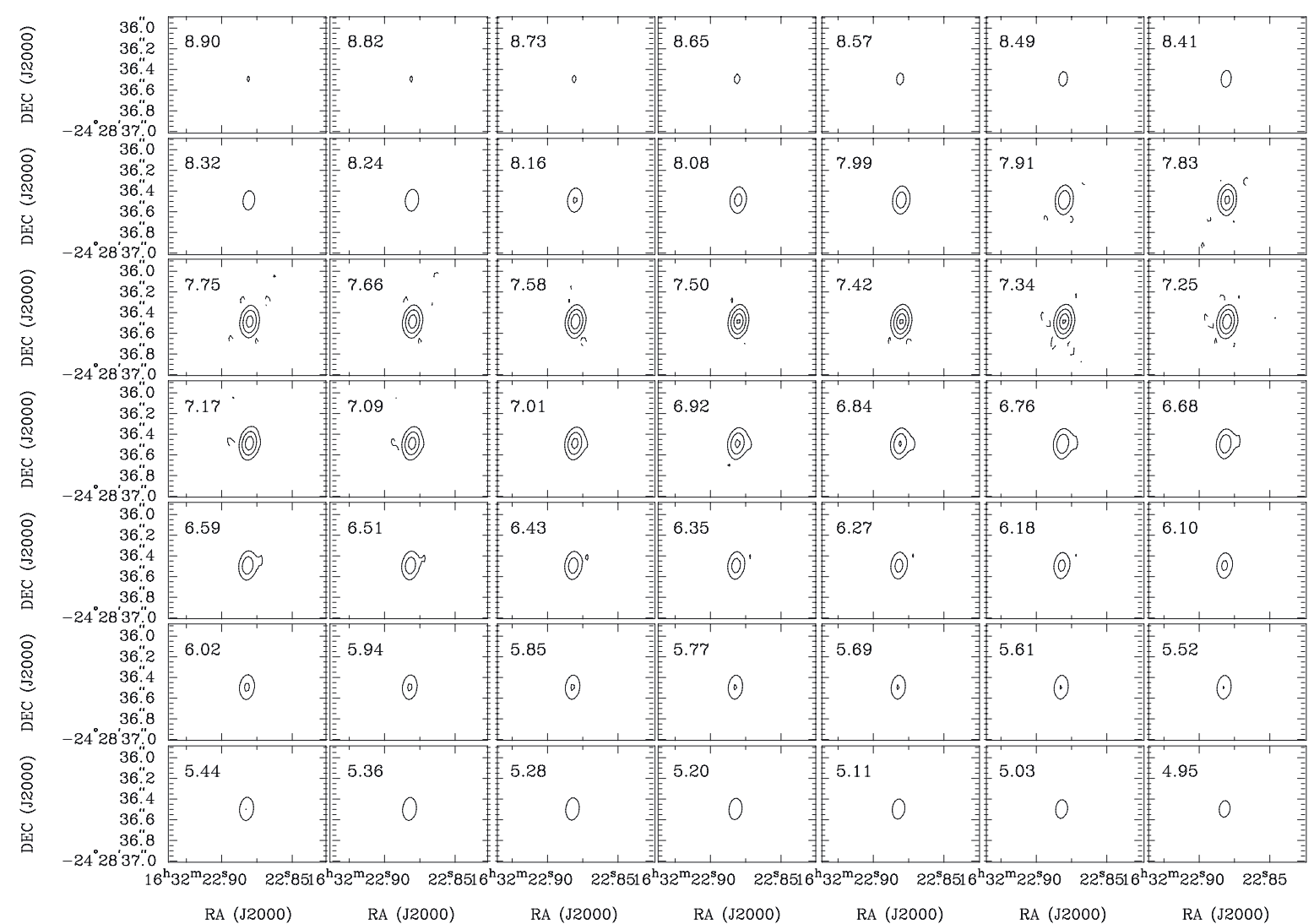

Fig. 1. Channel maps of the $\mathrm{H}_{2} \mathrm{O}$ emission toward IRAS $16293-2422$. Contours are $-50,50,500,3 \times 10^{3}, 1 \times 10^{4}, 2 \times 10^{4}$ times 8 mJy beam ${ }^{-1}$, the rms noise of the maps with lower intensities (see Sect. 2). The peak flux of $167 \mathrm{Jy} \mathrm{beam}^{-1}$ occurs at $V_{\mathrm{LSR}}=7.4 \mathrm{~km} \mathrm{~s}^{-1}$. The LSR velocity of each channel is labeled.

A scheme of the distribution of dust and molecular outflows in I16293 is shown in Fig. 2. The masers detected in our observations are associated with source A. They are located $\sim 0.25^{\prime \prime} \quad(\sim 30 \mathrm{AU}$ in projection) to the southeast of the dust condensation $A a$ resolved by the subarcsecond submillimeter (submm) observations of Chandler et al. (2005).

The Stokes $I$ spectrum of the maser emission is shown in Fig. 3 (upper panel). The non-Gaussian line profile indicates that there are unresolved components in the spectrum. Apart from the peak intensity at $7.4 \mathrm{~km} \mathrm{~s}^{-1}$, unresolved emission also seems to be present at lower velocities $\left(V_{\mathrm{LSR}} \simeq\right.$ $5.5 \mathrm{~km} \mathrm{~s}^{-1}$ ) with a strong flux of $\sim 20 \mathrm{Jy} \mathrm{beam}^{-1}$. Fainter emission $\left(\sim 5 \mathrm{Jy} \mathrm{beam}^{-1}\right)$ is also observed at higher velocity channels $\left(V_{\mathrm{LSR}} \simeq 9.2 \mathrm{~km} \mathrm{~s}^{-1}\right)$. Therefore, there are at least three unresolved components (Table 1). A two-dimensional Gaussian profile fit to each of those components provides a mean spatial separation of $\sim 22$ milli-arcsec (mas), which is larger than the precision in the relative position determination $\left(\mathrm{rms}_{\text {pos }} \simeq 2\right.$ mas, as estimated from the ratio of the width of the synthesized beam to the signal-to-noise ratio of the fainter component).

\subsection{Polarized emission}

The spectrum of linearly polarized intensity is very similar to the Stokes $I$ line profile except for the flux scale. It peaks at the same systemic velocity as the Stokes $I$ spectrum, but is weaker by a factor of $\sim 30$. The dependence of the linear polarization intensity, polarization fraction, position angle, and Stokes I parameter with systematic velocity is shown in Fig. 3 . The measured polarization fraction is $2.5 \pm 0.2 \%$. The polarization position angle $\theta$ is $-23^{\circ}$ and shows only small changes across the maser $\left(\sigma_{\theta}=2^{\circ}\right)$,
Table 1. $\mathrm{H}_{2} \mathrm{O}$ maser components in IRAS 16293-2422.

\begin{tabular}{lccc}
\hline \hline $\begin{array}{l}V_{\mathrm{LSR}} \\
\left(\mathrm{km} \mathrm{s}^{-1}\right)\end{array}$ & $\begin{array}{c}I_{\text {peak }}{ }^{a} \\
\left(\mathrm{Jy} \mathrm{beam}^{-1}\right)\end{array}$ & $\begin{array}{c}\alpha(2000) \\
(\mathrm{h} \mathrm{m} \mathrm{s})\end{array}$ & $\begin{array}{c}\delta(2000) \\
\left({ }^{\prime}{ }^{\prime \prime}\right)\end{array}$ \\
\hline 5.7 & 23 & 163222.8830 & -242836.495 \\
7.4 & 168 & 163222.8808 & -242836.487 \\
9.2 & 5 & 163222.8818 & -242836.493 \\
\hline
\end{tabular}

Notes. ${ }^{(a)}$ Equatorial coordinates derived with the JMFIT task of AIPS.

implying that the polarization vectors at different velocities trace basically the same region. The formal uncertainty in PA $\left(\sigma_{\theta}=\right.$ $\frac{1}{2} \frac{\sigma_{P}}{I_{P}} \frac{180^{\circ}}{\pi}$, Wardle \& Kronberg 1974) is small and ranges between $\sim 0.14^{\circ}$ and $0.80^{\circ}$ considering channels with strong and weak linear polarization emission, respectively. The linear polarization as derived from the Stokes $U$ and $Q$ maps is exhibited in Fig. 4, which shows the distribution of polarization vectors in the brightest velocity channel. The peak of the polarized intensity is offset by $0.05^{\prime \prime}$ from the Stokes $I$ peak. The polarization vectors may be either parallel or perpendicular to the magnetic field orientation in the plane of the sky. In Sect. 5, we discuss any assumptions that must be considered in order to solve this ambiguity.

The line profile of the circular polarization (Stokes $V$ ) has the characteristic S-shape (see Fig. 5, lower panel). The Stokes $V$ spectrum is proportional to both the first derivative of the Stokes $I$ spectrum and the LOS component of the magnetic field. The red dashed line in Fig. 5 indicates the fit that most closely represents this derivation. Owing to the remaining gain differences among the two polarizations, scaled-down replicas of the Stokes $I$ spectrum might contaminate the Stokes $V$ data 


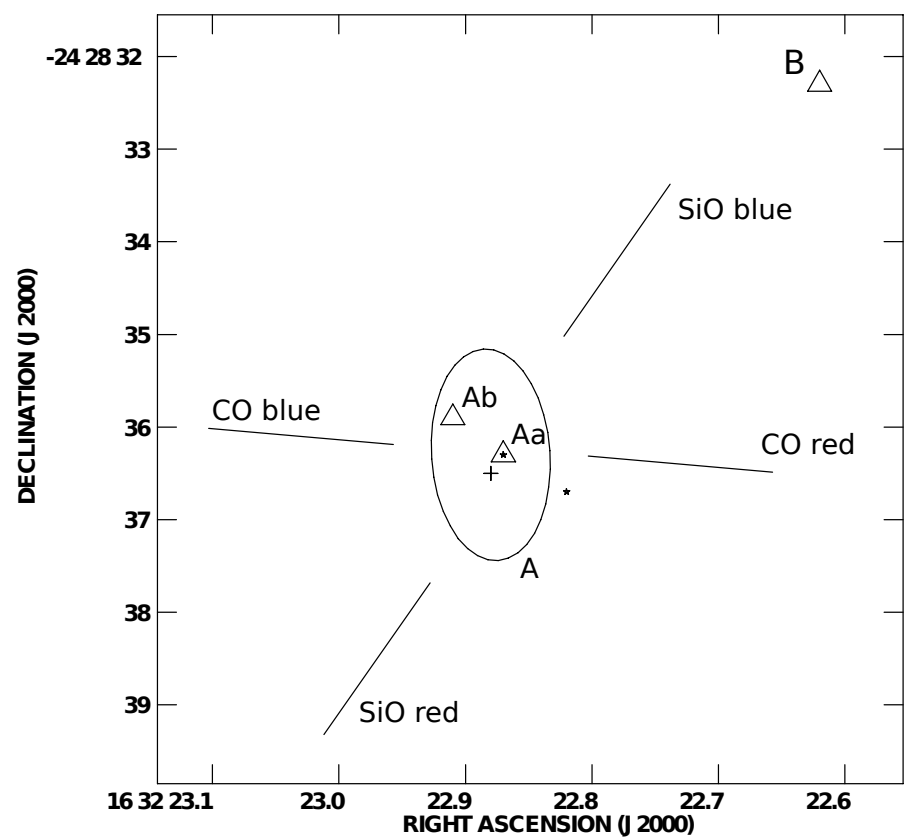

Fig. 2. Scheme of the distribution of dust and molecular material in I16293. The plus signal indicates the position of the peak intensity of our VLA water maser data. The ellipse is the deconvolved size of the dust continuum source A as derived by Rao et al. (2009). Triangles denote the position of the submillimeter condensations observed by Chandler et al. (2005). Stars denote the VLBI water maser detections of Imai et al. (2007) and straight lines denotes the direction of the CO and $\mathrm{SiO}$ outflows associated with this core (Rao et al. 2009).

(Sarma et al. 2001; Vlemmings et al. 2002). These features were considered in the fit and subtracted from the synthetic $V$ spectrum. The fraction of circular polarization, calculated as $\left(V_{\max }-V_{\min }\right) / I_{\max }$, is $\sim 0.45 \%$ for the brightest component. The remaining maser features show only residual Zeeman profiles with amplitudes at the rms level.

\section{4. $\mathrm{H}_{2} \mathrm{O}$ masers and the $\mathrm{SiO} / \mathrm{CO}$ outflows}

Figure 6 shows that the three possible maser features unresolved in our data are distributed linearly. The velocity shift observed between $V_{\mathrm{LSR}} \simeq 5.7 \mathrm{~km} \mathrm{~s}^{-1}$ and higher velocities is oriented in a $\mathrm{E}-\mathrm{W}$ direction $\left(\mathrm{PA} \simeq 110^{\circ}\right)$. The proximity to source $A a$ and the complex outflow configuration observed in this zone suggests that the maser emission is being pumped into the dense circumstellar material around this object.

Rao et al. (2009) reported a strong and very young SiO outflow extending toward the northwest-southeast (NW-SE) direction, with the SE lobe detected at redshifted LSR velocities (see Fig. 2). This SE component is also traced by a CO (3-2) outflow at $V_{\mathrm{LSR}} \simeq 9 \mathrm{~km} \mathrm{~s}^{-1}$ (Fig. 7 in Rao et al. 2009). The CO outflow has a dominant E-W orientation and has previously been reported in the literature (Yeh et al. 2008). Rao et al. (2009) claim that the $\mathrm{SiO}$ and $\mathrm{CO}$ outflows are centered on sources $A b$ and $A a$, respectively. Nevertheless, it is hard to identify the powering sources in their molecular maps, since the separation between $A a$ and $A b\left(\sim 0.6^{\prime \prime}\right)$ is much smaller than the HPBW of their $\mathrm{SiO}$ and $\mathrm{CO}$ maps $\left(\sim 3^{\prime \prime}\right)$.

Our maser features are also detected at redshifted velocities and lie in a similar direction to the $\mathrm{CO}$ outflow. On the other hand, their positions south of $A a$ suggest that they may be excited by the $\mathrm{SiO}$ outflow. The SE lobes observed for

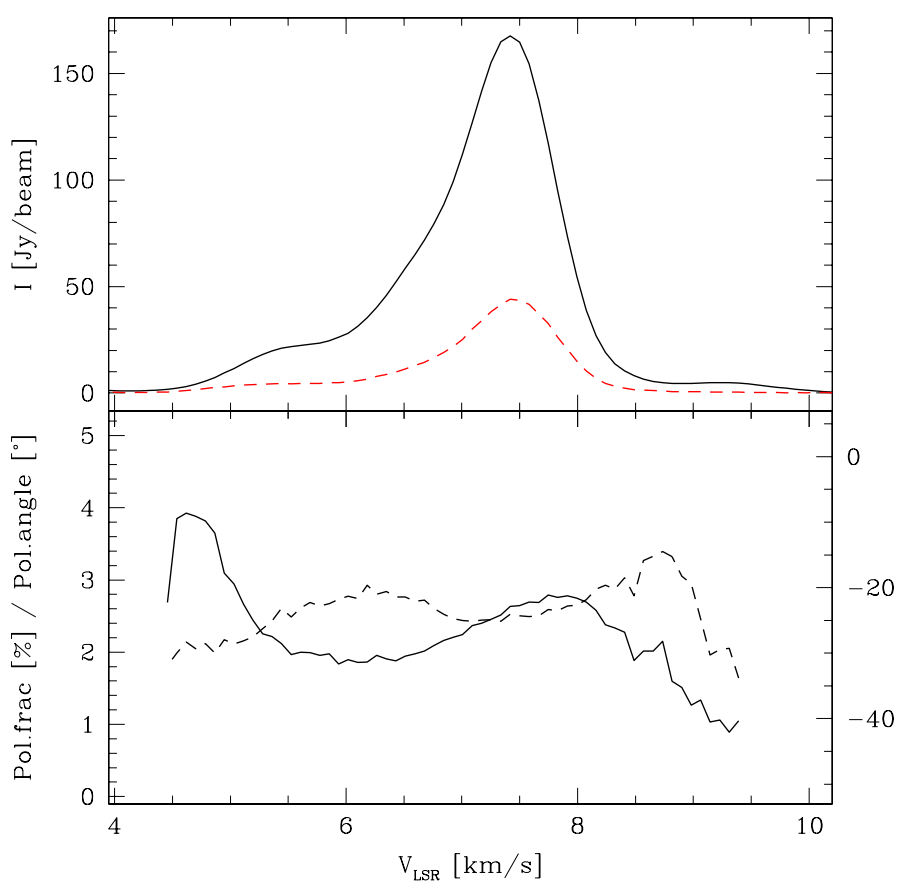

Fig. 3. Stokes $I$ (black line) and linear polarization intensity (multiplied by a factor of ten, red dashed line) spectra of the water maser emission (upper panel). The spectra of polarization fraction (black line, left scale) and position angle (dashed line, right scale) are also shown (lower panel). (A color version of this figure is provided in the online version.)

both $\mathrm{SiO}$ and $\mathrm{CO}$ at redshifted velocities (Figs. 6 and 7 from Rao et al. 2009) provide a close match to the LSR of our highest velocity features $\left(V_{\mathrm{LSR}} \sim 9 \mathrm{~km} \mathrm{~s}^{-1}\right)$. In addition, the VLA $3.7 \mathrm{~cm}$ data from Chandler et al. (2005) show two sources, A1 and A2, close to $A a$ (see Fig. 6). These authors refer to A2 as a protostar that powers a radio jet because of the bipolar shape seen in their high-resolution maps. On the other hand, source A1 would be an ionized region produced by shocks between this flow and nearby dense gas. Therefore, it is possible that the water masers are generated in the interaction regions traced by the radio sources and the outflows.

Water masers detections have also been reported toward source A through very long baseline (VLBI) observations (Imai et al. 2007). The milliarcsecond resolution data revealed a spot exactly over $A a$ and another one to the SW of it (see Fig. 2). While the former might have been excited in the circumstellar gas of source $A a$, the latter is likely to have been created by shocks in the E-W outflow. The redshifted lobe of this emission has an open shell structure, hence we expect to find masers that are offset away from the outflow main axis.

\section{The line-of-sight magnetic field strength in I16293}

From the Zeeman splitting formalism, the magnetic field strength can be correlated to the fraction of circular polarization by (see, for instance, Fiebig \& Guesten 1989)

$$
\begin{aligned}
P_{V} & =\left(V_{\max }-V_{\min }\right) / I_{\max } \\
& =2 \times A_{F-F^{\prime}} \times(B \cos \theta) / \Delta V_{I},
\end{aligned}
$$

where the $A_{F-F^{\prime}}$ coefficient depends on the maser rotational levels $F$ and $F^{\prime}$, the intrinsic thermal linewidth $\Delta v_{\text {th }}$, and the maser saturation degree, while $\Delta V_{I}$ is the full width at half maximum of the total power spectrum. For the $A_{F-F^{\prime}}$ coefficient, we 


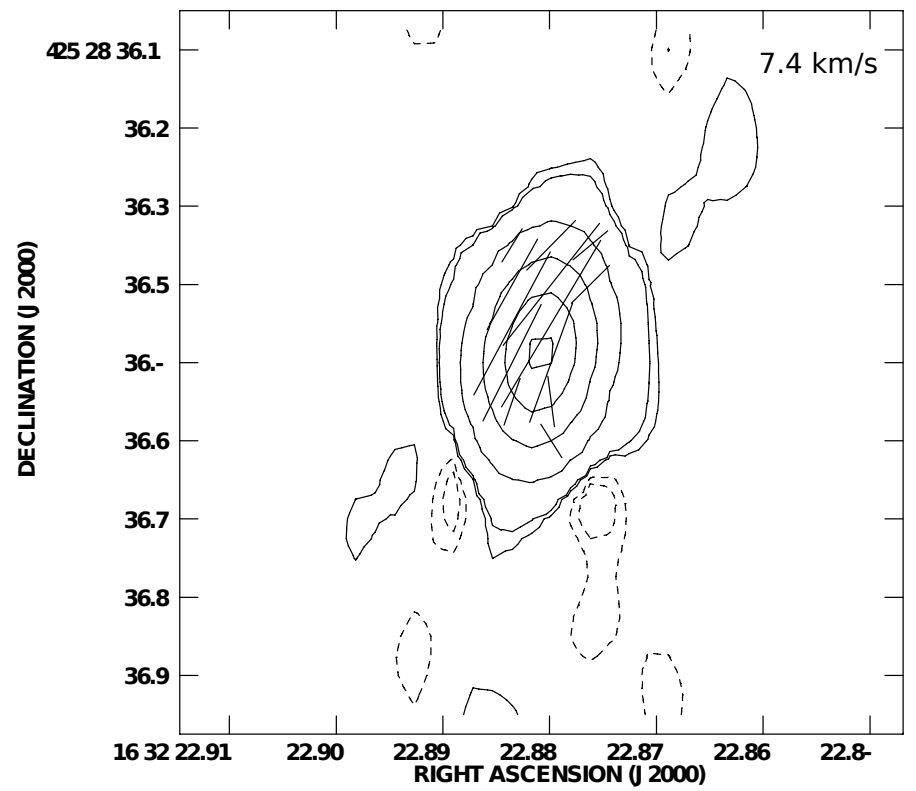

Fig. 4. Distribution of $\mathrm{H}_{2} \mathrm{O}$ linear polarization vectors in the brightest emission channel $\left(V_{\mathrm{LSR}} \simeq 7.4 \mathrm{~km} \mathrm{~s}^{-1}\right)$. Contours are $-50,-30,30,50$, $500,3 \times 10^{3}, 1 \times 10^{4}, 2 \times 10^{4} \times 8 \mathrm{mJy}$ beam $^{-1}$. Only polarization vectors whose $P>1 \%$ are plotted. Vectors are sampled as $1 / 2$ of a beam.

adopted the range of 0.012 to 0.018 , since these values are consistent with models and observations (Nedoluha \& Watson 1992; Vlemmings et al. 2002). The water maser spectrum is a blend of different velocity components, hence we adopt a linewidth ranging from $0.75 \mathrm{~km} \mathrm{~s}^{-1}$, which is typical of those found in other regions (Vlemmings et al. 2006), to $1.0 \mathrm{~km} \mathrm{~s}^{-1}$, the maximum estimated value in I16293 from our spectral profile. For these values, we find that $B_{\mathrm{LOS}}$ ranges from $\sim-94$ to $\sim-188 \mathrm{mG}$. The negative signal is inferred from the Stokes $V$ shape, meaning that the field is pointing toward the observer. For narrower linewidths $\left(\sim 0.75 \mathrm{~km} \mathrm{~s}^{-1}\right)$, which would be more realistic for resolved water maser lines, the field strength ranges from $\sim-94$ to $\sim-141 \mathrm{mG}$ (see Table 2). We note, however, that since the linear polarization fraction is about $3 \%$, this maser is likely unsaturated and the field strength determination is a fair approximation.

Figure 4 shows the linear polarization vectors at the velocity channel with the brightest emission. There is a degeneracy between the position angle of the linear polarization vectors and the magnetic field direction projected onto the plane-of-sky (POS). The position angle of the polarization is either parallel or perpendicular to the magnetic field in the POS for $\theta>\theta_{\text {crit }}$ or $\theta<\theta_{\text {crit }}=55^{\circ}$, respectively (Goldreich et al. 1973), where $\theta$ is the angle between $B$ and the maser propagation direction and $\theta_{\text {crit }}$ is the so-called Van Vleck angle. Given that the linear polarization fraction $P_{1}$ depends on $\theta$ and the maser saturation level, we should be able in principle to constrain the value of $\theta$ from our data and solve for this ambiguity. We used a non-local thermodynamic equilibrium radiative transfer model in order to fit the observed intensity $I$ and polarization fraction $P_{1}$. However, since our line is a blend of features that were spatially unresolved, we were unable to determine $\theta$. Although observations at more extended configurations would be needed to achieve this goal, we can at least claim that the POS field topology is quite ordered in both cases, i.e., it would remain ordered if rotated by $90^{\circ}$.

According to the submm data of Rao et al. (2009), the direction of the dust polarization vectors associated with source $\mathrm{A}$ averages to $95^{\circ}$, which implies that the POS magnetic field lines

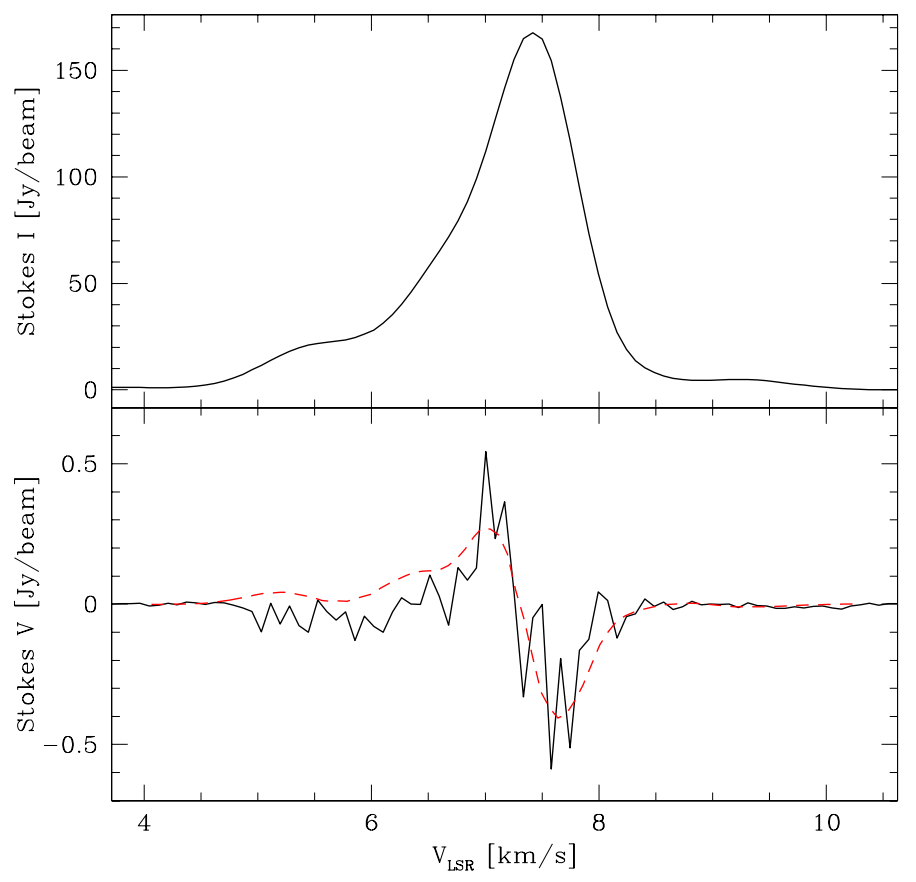

Fig. 5. Stokes I (upper panel) and Stokes $V$ (lower panel) spectra of the water maser emission. The red dashed line is the scaled derivative of the total power $I$ in order to guide the eye. (A color version of this figure is provided in the online version.)

have a PA of $5^{\circ}$ with respect to the north direction. Assuming that the $22 \mathrm{GHz}$ polarization vectors in Fig. 4 are parallel to the POS magnetic field, the main direction of the field lines is $-23^{\circ}$ (or its supplement, $157^{\circ}$ ) with respect to the north direction, as reported in Sect. 3.2. On the other hand, if our polarization vectors were perpendicular to the POS field, their main direction would then be $67^{\circ}$. Although two distinct scales are being compared, it is interesting to point out that in both cases there is clearly a change in the direction of the field lines on the small scales traced by our VLA data. In the case of parallel field lines and polarization vectors, such a change could be produced by the interaction of the $\mathrm{SiO}$ outflow and the magnetic field, given that the field lines lie almost in the same direction as the outflow (PA $\sim 145^{\circ}$, Fig. 2).

\section{Preshock magnetic fields and post-shock densities}

The only previous estimation of the magnetic field strength toward I16293 had been performed by Claussen et al. (2003), who used VLBI observations of water masers to estimate $B_{\mathrm{LOS}} \sim$ $-40 \mathrm{mG}$. Their maser spots appear to be associated with the A1 cm source (Fig. 6), which lies at a projected separation of $\sim 20 \mathrm{AU}$ at $V_{\mathrm{LSR}} \sim 2.5 \mathrm{~km} \mathrm{~s}^{-1}$ (Claussen, priv. comm.). Since this LSR velocity is similar to the core ambient velocity, this emission is unlikely to trace the same region as our VLA data. In any case, our study is one of the first determinations of the field strength in a low-mass young stellar object at densities higher than $10^{8} \mathrm{~cm}^{-3}$. As discussed in Sect. 4, the observed maser emission is excited in zones of compressed gas produced by shocks between the outflows and the ambient gas. In an ionized medium, shocks compress the gas by a factor $m_{\mathrm{A}}$, the Alfvénic Mach number that is defined as $v_{\mathrm{S}} / v_{\mathrm{A}}$, where $v_{\mathrm{S}}$ is the shock velocity and $v_{\mathrm{A}}$ the Alfvén speed. The magnetic field $B_{0}$ in the pre-shock zone is also enhanced by a factor $m_{\mathrm{A}}$ in the shocked 


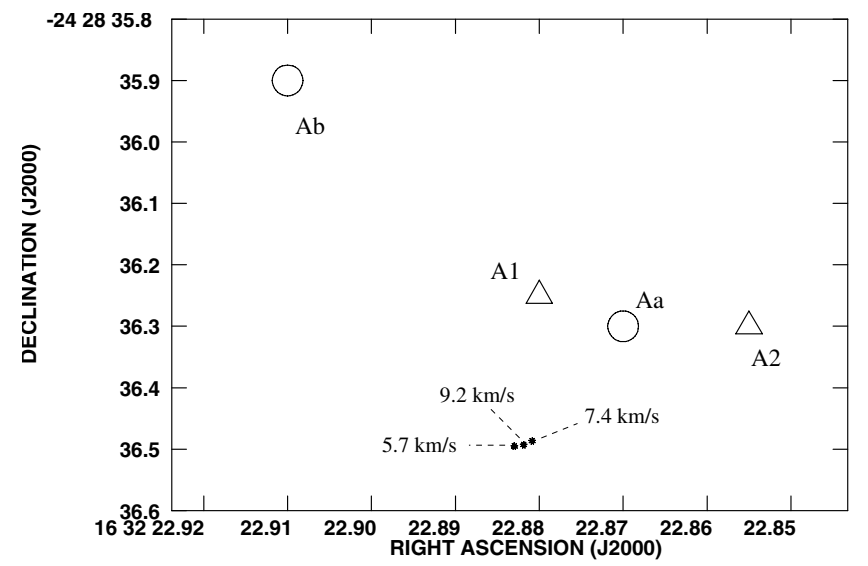

Fig. 6. Possible independent maser features (stars) derived using Gaussian fits. The accuracy in the position of each maser feature is $\sim 2$ mas, while the positions of the submm and $\mathrm{cm}$ sources are accurate to within $\sim 25$ mas and $\sim 10$ mas, respectively. The numerical labels indicate the maser velocities with respect to the local standard of rest (LSR). The $3.7 \mathrm{~cm}$ sources A1 and A2 (triangles) and the submm sources $A a$ and $A b$ (circles) are also shown (Chandler et al. 2005). The sizes of the symbols are not proportional to either their deconvolved sizes or their intensities.

Table 2. $B$-field strength calculation from Eq. (2) (assuming $\Delta V_{I}=$ $0.75 \mathrm{~km} \mathrm{~s}^{-1}$ ).

\begin{tabular}{cccc}
\hline \hline & $A_{F-F^{\prime}}=0.012$ & $A_{F-F^{\prime}}=0.015$ & $A_{F-F^{\prime}}=0.018$ \\
\hline$B_{\mathrm{LOS}}(\mathrm{mG})$ & -141 & -113 & -94 \\
\hline
\end{tabular}

gas so that $B_{\mathrm{s}} \sim m_{\mathrm{A}} \times B_{0}$. Since $v_{\mathrm{A}}$ varies with $B_{0}(n)^{-1 / 2}$, then $B_{\mathrm{s}}$ is proportional to $v_{\mathrm{s}}(n)^{1 / 2}$, where $n$ is the volume density of the pre-shock region. Rearranging this expression, the pre-shock density can be written as (Kaufman \& Neufeld 1996)

$n_{0}=1.6 \times 10^{6}\left(\frac{B_{\mathrm{s}}}{\mathrm{mG}}\right)^{2}\left(\frac{v_{\mathrm{s}}}{\mathrm{km} \mathrm{s}^{-1}}\right)^{-2} \mathrm{~cm}^{-3}$.

The LOS magnetic field strength calculated in Sect. 5 can be rescaled in order to obtain the total field strength $B_{\text {tot }}=B_{\mathrm{s}}$ in the shocked zone. According to Crutcher et al. (1999), the squared LOS magnetic field $\left\langle B_{\mathrm{LOS}}\right\rangle^{2}$ averaged over several lines of sight in the Galaxy is $\sim B_{\text {tot }}^{2} / 3$. The shock velocities are retrieved from submm water emission detected in shocked zones around I16293 (Ristorcelli et al. 2005). These data were modeled as C-shocks (low velocity, non-dissociative shocks, Kaufman \& Neufeld 1996) with shock velocities of $12-15 \mathrm{~km} \mathrm{~s}^{-1}$. Applying these values to Eq. (3), we obtain pre-shock densities of $\sim 4.2_{-2.4}^{+2.3} \times$ $10^{8} \mathrm{~cm}^{-3}$, which is consistent with the typical densities at which water masers are eventually pumped. The upper/lower limits to the number density refer to the $B_{\mathrm{s}}$ determinations for the two limiting $A_{F-F^{\prime}}$ cases (Table 2).

If the magnetic field dominates the outflow dynamics, the magnetic pressure should be similar to the shock ram pressure, i.e.,

$$
B_{\mathrm{s}}^{2} / 8 \pi=\rho_{0} v_{\mathrm{s}}^{2},
$$

where $\rho_{0}$ is the pre-shock density. For the field strength $B_{s}$ determined in the previous section and shock velocities of Ristorcelli et al. (2005), we find a mean pre-shock density of $\sim 2 \times 10^{8} \mathrm{~cm}^{-3}$, which is consistent with the pre-shock densities calculated from Eq. (3) and in line with a magnetically controlled outflow evolution.
Assuming magnetic flux-freezing, the compression of an ordered magnetic field $\left(B_{0}\right)$ amplifies the field strength in proportion to the gas density according to

$\frac{B_{0}}{n_{0}}=\frac{B_{\mathrm{s}}}{n_{\mathrm{s}}}$,

where $n_{\mathrm{s}}$ is the post-shock density. If we consider that the shock compresses the well-established field geometry in the submm maps of Rao et al. (2009), which predict a (scaled) $B_{0} \simeq 9 \mathrm{mG}$ at densities $n_{0} \simeq 4.9 \times 10^{7} \mathrm{~cm}^{-3}$, and using the post-shock field intensity $B_{\mathrm{s}}$ calculated from our data, we find post-shock densities of $\sim 1.3 \times 10^{9} \mathrm{~cm}^{-3}$, which is expected for effective water maser pumping. In a more conservative approach, we rescale the magnetic field strength of Rao et al. (2009) using $B \propto n^{\kappa}$, where $\kappa$ may have distinct values depending on the dynamics of the core: $\kappa=0.5$ for a cloud evolution controlled by the magnetic field (Crutcher et al. 1999), $\kappa=0.47$, in the case of magnetic evolution as numerically modeled by Fiedler \& Mouschovias (1993), and $\kappa=0.67$ for a free-fall cloud collapse. For the three cases, the pre-shock magnetic field in the calculated pre-shock densities ranges between $\sim 24$ and $36 \mathrm{mG}$. Applying again Eq. (5), we obtain post-shock densities in the range $\sim 2-5 \times 10^{9} \mathrm{~cm}^{-3}$, which are again consistent with typical $\mathrm{H}_{2} \mathrm{O}$ maser densities, but we are unable to discern in which dynamical regime, if magnetic or turbulent, the core evolves.

The $\mathrm{H}_{2} \mathrm{O}$ maser emission of $\mathrm{I} 16293$ is strong (>200 Jy) and stable over a few weeks but highly variable over months, as shown by surveys performed toward this source (Wilking \& Claussen 1987; Claussen et al. 1996; Furuya et al. 2003). We therefore hope to carry out VLBI observations in the future. With these observations, the blended maser components found in this work will likely be resolved both spatially and spectroscopically. We will then be able to apply radiative transfer models similar to those in Vlemmings (2006) to derive the full 3-D magnetic field properties as has already been done in other star-forming regions (e.g., Cepheus A HW2, Vlemmings et al. 2006).

\section{Conclusions}

We have reported on the $\mathrm{H}_{2} \mathrm{O}\left(6_{16}-5_{23}\right)$ maser emission observed with the VLA/EVLA toward the low-mass source IRAS 16293-2422. This is one of the first estimations of the lineof-sight magnetic field strength in a low-mass protostar at densities higher than $10^{8} \mathrm{~cm}^{-3}$. From this study, we have obtained insights into the dynamics of this object at such high densities. Our main conclusions are:

- We detect strong water-maser emission associated with the submm source $A a$.

- The maser spectrum has a non-Gaussian profile, which is indicative of at least three maser features covering a velocity range of $\sim 3.5 \mathrm{~km} \mathrm{~s}^{-1}$ distributed in a linear configuration. These components are likely excited in zones of compressed gas produced by shocks in the outflow activity of I16293.

- The obtained Stokes $V$ spectrum is consistent with Zeeman emission. The mean LOS magnetic field strength is $\sim 113 \mathrm{mG}$. The POS field topology retrieved from the linear polarimetry shows an ordered pattern consistent with larger-scale field morphologies.

- The post- and pre-shock densities calculated from our field strength estimation are consistent with the typical densities expected for $\mathrm{H}_{2} \mathrm{O}$ maser pumping. 
- The dynamics of the outflow evolution in these sources are likely regulated by the magnetic field, since the magnetic pressure in the shocked gas is similar to the pre-shock ram pressure.

Acknowledgements. The authors would like to thank the anonymous referee for a constructive report that helped to improve the paper. We are also grateful to Gabriele Surcis for running the radiative transfer models on the maser data. This research was partially supported by the Deutsche Forschungsgemeinschaft (DFG) through the Emmy Noether Research grant VL 61/3-1 and through SFB 956. F.O.A., J.M.G., and J.M.T. also acknowledge support from MICINN (Spain) AYA2011-30228-C03 and AGAUR (Catalonia) 2009SGR1172 grants.

\section{References}

Bisschop, S. E., Jørgensen, J. K., Bourke, T. L., Bottinelli, S., \& van Dishoeck, E. F. 2008, A\&A, 488, 959

Briggs, D. 1995, Ph.D. Thesis, The New Mexico Institute of Mining and Technology

Ceccarelli, C., Loinard, L., Castets, A., Tielens, A. G. G. M., \& Caux, E. 2000, A\&A, 357, L9

Chandler, C. J., Brogan, C. L., Shirley, Y. L., \& Loinard, L. 2005, ApJ, 632, 371

Claussen, M. J., Wilking, B. A., Benson, P. J., et al. 1996, ApJS, 106, 111

Claussen, M., Sarma, A. P., Wootten, A., Marvel, K. B., \& Wilking, B. 2003, in Meeting Abstracts, BAAS, 35, 1362

Crutcher, R. M., Roberts, D. A., Troland, T. H., \& Goss, W. M. 1999, ApJ, 515, 275

Elitzur, M., Hollenbach, D. J., \& McKee, C. F. 1989, ApJ, 346, 983

Fiebig, D., \& Guesten, R. 1989, A\&A, 214, 333

Fiedler, R. A., \& Mouschovias, T. C. 1993, ApJ, 415, 680

Furuya, R. S., Kitamura, Y., Wootten, A., Claussen, M. J., \& Kawabe, R. 2003, ApJS, 144, 71

Goldreich, P., Keeley, D. A., \& Kwan, J. Y. 1973, ApJ, 179, 111

Goodman, A. A., Jones, T. J., Lada, E. A., \& Myers, P. C. 1992, ApJ, 399, 108
Goodman, A. A., Jones, T. J., Lada, E. A., \& Myers, P. C. 1995, ApJ, 448, 748 Imai, H., Nakashima, K., Bushimata, T., et al. 2007, PASJ, 59, 1107

Jørgensen, J. K., Bourke, T. L., Nguyen Luong, Q., \& Takakuwa, S. 2011, A\&A, 534, A100

Kaufman, M. J., \& Neufeld, D. A. 1996, ApJ, 456, 250

Kuan, Y., Huang, H., Charnley, S. B., et al. 2004, ApJ, 616, L27

Lazarian, A., Goodman, A. A., \& Myers, P. C. 1997, ApJ, 490, 273

Loinard, L., Torres, R. M., Mioduszewski, A. J., \& Rodríguez, L. F. 2008, ApJ, 675, L29

Looney, L. W., Mundy, L. G., \& Welch, W. J. 2000, ApJ, 529, 477

Mundy, L. G., Wootten, H. A., \& Wilking, B. A. 1990, ApJ, 352, 159

Nedoluha, G. E., \& Watson, W. D. 1992, ApJ, 384, 185

Pérez-Sánchez, A. F., Vlemmings, W. H. T., \& Chapman, J. M. 2011, MNRAS, 418,1402

Rao, R., Girart, J. M., Marrone, D. P., Lai, S., \& Schnee, S. 2009, ApJ, 707, 921

Ristorcelli, I., Falgarone, E., Schöier, F., et al. 2005, in IAU Symp., 235, 227P

Sanna, A., Moscadelli, L., Cesaroni, R., et al. 2010a, A\&A, 517, A71

Sanna, A., Moscadelli, L., Cesaroni, R., et al. 2010b, A\&A, 517, A78

Sarma, A. P., Troland, T. H., \& Romney, J. D. 2001, ApJ, 554, L217

Stark, R., Sandell, G., Beck, S. C., et al. 2004, ApJ, 608, 341

Surcis, G., Vlemmings, W. H. T., Curiel, S., et al. 2011a, A\&A, 527, A48

Surcis, G., Vlemmings, W. H. T., Torres, R. M., van Langevelde, H. J., \& Hutawarakorn Kramer, B. 2011b, A\&A, 533, A47

Tamura, M., Hayashi, S. S., Yamashita, T., Duncan, W. D., \& Hough, J. H. 1993, ApJ, 404, L21

Terebey, S., Vogel, S. N., \& Myers, P. C. 1992, ApJ, 390, 181

Vlemmings, W. H. T. 2006, A\&A, 445, 1031

Vlemmings, W. H. T. 2008, A\&A, 484, 773

Vlemmings, W. H. T., Diamond, P. J., \& van Langevelde, H. J. 2002, A\&A, 394, 589

Vlemmings, W. H. T., Diamond, P. J., van Langevelde, H. J., \& Torrelles, J. M. 2006, A\&A, 448, 597

Walker, C. K., Lada, C. J., Young, E. T., \& Margulis, M. 1988, ApJ, 332, 335

Wardle, J. F. C., \& Kronberg, P. P. 1974, ApJ, 194, 249

Wilking, B. A., \& Claussen, M. J. 1987, ApJ, 320, L133

Wootten, A. 1989, ApJ, 337, 858

Yeh, S. C. C., Hirano, N., Bourke, T. L., et al. 2008, ApJ, 675, 454 